\title{
Comparative Evaluation of Widal Slide Agglutination Test and Widal Tube Agglutination Test in Diagnosing Enteric Fever among Patients Attending a Tertiary Care Hospital in Western Odisha
}

\author{
Shuvankar Mukherjee ${ }^{1}$, Anshuman Dash², Shreekant Tiwari³
}

${ }^{1}$ Department of Microbiology, Hitech Medical College, Rourkela, Odisha, India. ${ }^{2}$ Department of Microbiology, Hitech Medical College, Rourkela, Odisha, India. ${ }^{3}$ Department of Microbiology, Hitech Medical College, Rourkela, Odisha, India.

\section{ABSTRACT}

\section{BACKGROUND}

Enteric fever is a feco-orally transmitted disease with significant morbidity and mortality in India. Isolation of responsible bacteria from blood or bone marrow is the gold standard method for Enteric fever but it is seldom used now a days due to long turnaround time and higher cost. In developing country like India, relatively cheaper Widal test is used to diagnose Enteric fever. There are two methods of Widal test- slide agglutination test and tube agglutination test. The present study was done to compare efficacy of the slide agglutination test and tube agglutination in diagnosing enteric fever.

\section{METHODS}

An OPD and IPD based cross sectional study was conducted from May 2019 to Oct 2019. A total of 100 patients with clinical suspicion of Enteric fever were included in the study. $15 \mathrm{ml}$ blood was collected. Blood culture of all the samples were also performed by inoculating $10 \mathrm{ml}$ of blood into appropriate blood culture media. Serum was separated from rest of the blood and serum was tested by both slide agglutination test and Widal tube agglutination test.

\section{RESULTS}

Among 100 patients, slide agglutination test demonstrated positive result in $40(40 \%)$ samples and Widal tube agglutination test demonstrated positive result in 27 (27\%) samples only. Among 40 samples positive by slide test 12 samples were negative by blood culture test. The slide test had a sensitivity of $93.33 \%$, specificity of $82.86 \%$, positive predictive value of $70 \%$ and negative predictive value of $96.67 \%$ and tube test had a sensitivity of $86.67 \%$, specificity of $98.57 \%$ positive predictive value of $96.30 \%$ and negative predictive value of $94.52 \%$ considering blood culture as gold standard.

\section{CONCLUSIONS}

Due to higher sensitivity but poor specificity, slide Widal test should be used for rapid screening test and positive samples should be confirmed by Widal tube agglutination test.

\section{KEY WORDS}

Enteric Fever, Slide Test, Tube Test, Widal, Sensitivity
Corresponding Author: Shuvankar Mukherjee, Staff Quarter, Room No. 28, Hitech Medical College, Rourkela-769004, Odisha, India. E-mail: shrnu963@gmail.com

DOI: $10.14260 /$ jemds $/ 2020 / 2$

Financial or Other Competing Interests: None.

How to Cite This Article: Mukherjee S, Dash A, Tiwari S. Comparative evaluation of Widal slide agglutination test and Widal tube agglutination test in diagnosing enteric fever among patients attending a tertiary care hospital in Western Odisha. J. Evolution Med. Dent. Sci. 2020;9(01):5-9, DOI: $10.14260 /$ jemds $/ 2020 / 2$

Submission 04-11-2019, Peer Review 21-12-2019, Acceptance 28-12-2019, Published 06-01-2020. 


\section{BACKGROUND}

Enteric fever is a systemic disorder which includes both typhoid and paratyphoid fever. According to WHO, incidence rate of typhoid is 21.5 million globally in the year $2000^{1}$ and in Asia alone it is 13 million. ${ }^{2}$ Typhoid fever is caused by Salmonella typhi and Paratyphoid fever is caused by Salmonella paratyphi A and B. Serotype C is very much rare in India. Patient usually acquire the infection from contaminated drinks and food and source is usually a chronic carrier or convalescent carrier. Poor sanitation, unhygienic food preparation, overcrowding all leads to over incidence of Enteric fever in developing country like India. Laboratory diagnosis is usually done by Blood/Bone marrow culture in $1^{\text {st }}$ week and by Widal test $2^{\text {nd }}$ week onwards. Although isolation of organism from blood/bone marrow or stool sample are more confirmative for diagnosis Widal test is still initial investigation of choice in India because of its low cost, short turnaround time and simplicity.3,4,5 Asymptomatic carrier acts as source of infection of enteric fever and mode of transmission is through contaminated drink or food. Food borne infection has shorter incubation period and higher attack rate compared to water borne transmission as food borne infection involves ingestion of large no of microorganism. Sexual route of transmission including by anal or oral sex has been reported but direct person-toperson transmission is not common. Doctors can acquire the infection from infected patients due to lack of hand hygiene or during laboratory work. Enteric fever is a health problem of developing world mainly. Annual prevalence of enteric fever is 21.6 million cases for typhoid and 5.5 million cases for paratyphoid. Incidence ranges from 25 to 1000 cases per 100,000 population in developing regions. ${ }^{6}$ Annual death rate is 200,000 to 600,000 in endemic region. ${ }^{6}$ South-central Asia and Southeast Asia are area of high incidence (100/100,000 cases/year). The rest of Asia, Africa, the Caribbean and South America, and Oceania, are area of medium incidence (10100/100,000 cases/year). ${ }^{6}$

The incidence of typhoid and paratyphoid is higher with region of poor sanitation and poor drinking water. Young children and 1 to 15 years old child are infected more than adults in urban area of endemic region. Risk factors are polluted water or ice, flood situation, food and water purchased from unhygienic shop or restaurant, raw vegetables and fruits contaminated with sewage, ill contacts with the patient in home, lack of hand and toilets hygiene, and Helicobacter pylori infected patient, who has decreased gastric acidity. Resistance to first line antibiotic chloramphenicol ampicillin and trimethoprim has been reported in many places of the world specially in south east Asia including India. ${ }^{6}$ These antibiotic resistant bacteria causes more complication specially in children of developing country. There are two methods available for Widal test; slide Widal test and tube Widal test. Both methods have their own merits and demerits but as slide agglutination test is cheaper, faster and easy to perform it is more popular test in developing country. ${ }^{5}$ But question arises upon reliability of slide agglutination test. Should we blindly start antibiotic if slide test is positive? If we blindly rely upon slide agglutination test in case of false positive, we are giving the patient unnecessary antibiotic that may lead to antibiotic resistance in future. So, in this era of antibiotic stewardship we should test reliability of slide agglutination test in comparison to tube test. Keeping it in mind we have conducted a research in a tertiary care hospital of Western Odisha to detect diagnostic efficacy of Widal slide test against Widal tube agglutination test in Enteric fever considering blood culture as gold standard.

\section{METHODS}

It was a cross sectional study involving patients of IPD and OPD, done during a period of 6 months from May 2019 to Oct 2019.

\section{Inclusion Criteria}

Patient of all age group and both sexes attending the inpatient and outpatient departments of Departments of Medicine and Paediatrics with clinical presentation suggestive of enteric fever i.e. continuous high fever for more than 7 days with abdominal discomfort with either constipation or diarrhoea, coated tongue, hepatosplenomegaly, relative bradycardia with or without rose spot.

\section{Exclusion Criteria}

Very severely ill patient suffering from other non-enteric disease and those who were found to be diagnosed with patients on antibiotic and those who were found to be diagnosed with other disease such as malaria hepatitis dengue etc., patients on antibiotic patient, who are recently vaccinated against typhoid and who have refused to give blood were excluded from the study.

\section{Study Subject}

Blood samples of 100 patients presenting features of enteric fever were collected and subjected to slide agglutination test, Widal tube agglutination test as well as blood culture in the Department of Microbiology.

\section{Sample Collection}

From each individual included in the study under strict aseptic precaution $15 \mathrm{ml}$ of venous blood was withdrawn. 10 $\mathrm{ml}$ inserted into brain heart infusion broth for blood culture and $5 \mathrm{ml}$ into a well labelled plain tube. Sera was separated with centrifugation (3000 rpm for 2 minutes) machine.

Sera were subjected to the slide agglutination test. The test was performed as per the manufacturer instruction (Span Diagnostic Ltd, India).One drop (50 microlitre) of undiluted test serum was placed on the circles of the slide provided in the kit along with positive control serum followed by addition of one drop of antigen $\mathrm{O}, \mathrm{H}, \mathrm{AH}$ and $\mathrm{BH}$. The contents were mixed with separate applicator stick and the slide was rocked gently for 1 minute. if no agglutination was observed the test was considered negative. Semiquantitative slide test was done in every positive sample to know the titer. Serial dilution of 50 units of serum was done with the help of normal saline $(1: 20,1: 40,1: 80,1: 160,1: 320)$ then one drop of antigen was put, and the slide was rotated for 2 minutes and highest dilution showing the agglutination was considered as titer. All the samples were subjected to 
tube agglutination test to confirm the result of slide agglutination test according manufacturer instruction with the serial dilution technique. $900 \mathrm{ul}$ of normal saline is mixed with $100 \mathrm{ul}$ of serum in first test tube then $500 \mathrm{ul}$ of this mixture was transferred to next test tube which was loaded with $500 \mathrm{ul}$ of normal saline (Consequently 5 tubes to make dilution 1:20, 1:40, 1:80, 1:160, 1:320). 500 ul of mixture was discarded from last test tube. One drop of antigen was mixed in every test tube and well mixed. With the help of Widal rack all mixture were put in water bath at 55-degree temperature for 18 hours and next day depending on highest dilution showing agglutination, titer were measured. Both in slide test and tube test titer more or equal to $1: 80$ for 0 agglutination test and 1:160 for $\mathrm{H} / \mathrm{AH} / \mathrm{BH}$ agglutination test considered positive depending on local titer. All 100 samples were subjected to blood culture by mixing $10 \mathrm{ml}$ of blood to brain heart infusion broth and subculturing to Blood agar and MacConkey agar. Salmonella species were identified by standard biochemical test (Indole, TSI, Citrate and motility test) and slide agglutination test. Data was analysed with SPSS software Ver. 20.

\section{RESULTS}

A total of 100 cases apparently suffering from enteric fever were enrolled in the study whose blood samples were tested serologically. Out of 100 samples slide agglutination test showed positive result in $40(40 \%)$ samples and negative result in $60(60 \%)$ samples (Table. 1). Out of 100 samples Widal tube agglutination test showed positive result in 27 (27\%) samples and negative result in 73 (73\%) samples (Table 1). Among all samples 30 (30\%) were positive by blood culture and $70(70 \%)$ were negative for blood culture(Table 1). Among 40 samples positive by slide test $62.5 \%(25 / 40)$ were positive by tube test and $37.5 \%(15 / 40)$ were negative by tube test. Among 60 samples negative by slide test $3.3 \%(2 / 60)$ were positive by tube test and $96.6 \%$ (58/60) were negative by tube test (Table 2$) .30 \%$ samples were positive for blood culture. Among 30 samples positive by blood culture 28 were successfully detected positive by slide test but tube test detected only 26 positives. (Table 3 \& $4)$.

\begin{tabular}{|c|c|c|c|}
\hline Test & Positive & Negative & Total \\
\hline Slide Widal & $40(40 \%)$ & $60(60 \%)$ & $100(100 \%)$ \\
\hline Tube Widal & $27(27 \%)$ & $73(73 \%)$ & $100(100 \%)$ \\
\hline Blood culture & $30(30 \%)$ & $70(70 \%)$ & $100(100 \%)$ \\
\hline \multicolumn{3}{|c|}{ Table 1. Positivity Rate of Slide Widal and Tube Widal } \\
and Blood Culture \\
\hline
\end{tabular}

\begin{tabular}{|c|c|c|c|}
\hline \multicolumn{4}{|c|}{ Tube Test } \\
\hline Slide Test & Positive & Negative & Total \\
\hline Positive & 25 & 15 & 40 \\
\hline Negative & 2 & 58 & 60 \\
\hline Total & 27 & 73 & 100 \\
\hline \multicolumn{4}{|c|}{ Table 2. Slide Test vs Tube Test } \\
\hline
\end{tabular}

\begin{tabular}{|c|c|c|c|}
\hline \multicolumn{4}{|c|}{ Blood Culture } \\
\hline Slide Test & Positive & Negative & Total \\
\hline Positive & 28 & 12 & 40 \\
\hline Negative & 2 & 58 & 60 \\
\hline Total & $\mathbf{3 0}$ & $\mathbf{7 0}$ & $\mathbf{1 0 0}$ \\
\hline \multicolumn{4}{|c|}{ Table 3. Slide Test vs Blood Culture } \\
\hline
\end{tabular}

\begin{tabular}{|c|c|c|c|}
\hline \multicolumn{4}{|c|}{ Blood Culture } \\
\hline Tube Test & Positive & Negative & Total \\
\hline Positive & 26 & 1 & 27 \\
\hline Negative & 4 & 69 & 73 \\
\hline Total & $\mathbf{3 0}$ & $\mathbf{7 0}$ & 100 \\
\hline \multicolumn{4}{|c|}{ Table 4. Tube Test vs Blood Culture } \\
\hline
\end{tabular}

\begin{tabular}{|c|c|c|}
\hline \multicolumn{2}{|c|}{ Slide Widal } & Tube Widal \\
\hline Sensitivity & $\mathbf{9 3 . 3 3 \%}$ & $86.67 \%$ \\
\hline Specificity & $82.86 \%$ & $\mathbf{9 8 . 5 7 \%}$ \\
\hline Positive predictive value & $70 \%$ & $96.30 \%$ \\
\hline Negative predictive value & $\mathbf{9 6 . 6 7 \%}$ & $\mathbf{9 4 . 5 2 \%}$ \\
\hline \multicolumn{2}{|c|}{ Table 5. Sensitivity and Specificity of Widal Test } \\
(Blood Culture as Gold Standard)
\end{tabular}

Similarly, among 70 samples negative by blood culture 69 were successfully declared negative by tube test but slide test showed negative only in 58 samples. (Table $3 \& 4$ ). The slide test had a sensitivity of $93.33 \%$, specificity of $82.86 \%$, positive predictive value of $70 \%$ and negative predictive value of $96.67 \%$ and tube test had a sensitivity of $86.67 \%$, specificity $98.57 \%$ positive predictive value $96.30 \%$ and negative predictive value of $94.52 \%$. (considering blood culture as gold standard) (Table 5)

\section{DISCUSSION}

Salmonellae are named for the pathologist Salmon, who first isolated Salmonella choleraesuis from porcine intestine 6 . Salmonella are effective commensals and pathogens that cause a spectrum of diseases in humans and animals, including domesticated and wild mammals, reptiles, birds, and insects. Some Salmonella serotypes, such as Salmonella Typhi, Salmonella Paratyphi, and Salmonella Sendai, are highly adapted to humans and have no other known natural hosts, whereas others, such as Salmonella Typhimurium, have a broad host range and can infect a wide variety of animal hosts and humans. Some Salmonella serotypes, such as Dublin (Cattle) and Arizonae (Reptiles), are mostly adapted to an animal species and only occasionally infect humans. The distribution of Salmonella in the environment is wide. Before the 19th century, typhus and typhoid fever were confused.

Though various clinical distinctions were proposed, none reliably distinguished these syndromes. In 1829 in Paris, P. Ch. A. Louis separated typhoid from other fevers on the basis of intestinal lymph node and spleen pathology ${ }^{6}$. He also described the clinical phenomena of rose spots, intestinal perforation, and haemorrhage. In the English literature, William Jenner in 1850 settled the question of whether typhus and typhoid were different diseases ${ }^{6}$. He distinguished typhoid based on the pathologic evidence of enlargement of the Peyer's patches and mesenteric lymph nodes. Jenner also noted that prior attacks of typhoid protected against subsequent attacks; this was not the case for typhus. In 1869, Wilson proposed the term enteric fever as an alternative to typhoid fever, given the anatomic site of infection. ${ }^{6}$ Though enteric fever remains a more accurate term, the use of the term typhoid persists today. Widal and others demonstrated that convalescent sera from typhoid patients caused the organisms to "stick together in large balls and lose their motility." Widal coined the term agglutinin to describe this observation. In contrast to other Salmonella 
serotypes, the etiologic agents of enteric fever-S. Typhi and S. Paratyphi serotypes A, B, and C have no known hosts other than humans.

In India Widal test is the most commonly used test to diagnose Enteric fever. It is advised after 1 week of fever to allow antibody to develop. In first week only method of diagnosis is blood culture which is $40-60 \%$ sensitive and gold standard. A background knowledge the local titer population may help in interpretation of the Widal test, and it should only be used in patients where there is a high suspicion (Prior Probability) of enteric fever. The Widal test ideally requires both an acute and a convalescent-phase serum sample taken approximately 10 days apart, and a positive result is determined by a fourfold rise in antibody titer. However, antibody titers in infected patients often rise before the clinical onset and patient often take prior antibiotic. So, it becomes difficult to demonstrate the required fourfold rise between initial and subsequent samples for a confirmatory diagnosis. In practice, the result of a single, acute phase serum sample is often used to start treatment although it is not scientifically sound.

Slide Widal sensitivity varies from $73-92 \%$ and specificity $72-82 \% .^{5}$ most of the earlier studies conducted worldwide revealed that the slide Widal test had high sensitivity (92\%) and tube Widal test had high specificity (100\%). In the present study slide Widal test showed high sensitivity $93.3 \%$ and high negative predictive value $96.67 \%$ which is similar to Oslen et $\mathrm{al}^{7}$ (2004) and Wilke et $\mathrm{al}^{8}$ (2002.). Karen H Keddy et $\mathrm{al}^{5}$ (2011) has reported the poor specificity and poor positive predictive value which is similar to our study (specificity $82.86 \%$ and PPV 70\%). In our study slide Widal test showed 40 samples positive for enteric fever. Among them 12 samples were declared negative by blood culture test. That means false positivity rate of slide agglutination test is very high $(30 \%)$ which is similar to study by Ayse wilke et $\mathrm{al}^{8}$ (2002) and Dr Jagdish C das et $\mathrm{al}^{9}$ (2007). Although Henry Welch et $\mathrm{al}^{10}$ (1939) and Handjo et al ${ }^{11}$ (2004) have reported that slide test is very much specific, it was not showed in our study. But tube test showed 27 positive and among these 27 only one was declared negative by blood culture. That means false positivity rate was very much low in case of tube test (3.7\%).

False positivity by slide agglutination test may be due cross reactivity with non-bacterial infection such as malaria, dengue, hepatitis $A$, and infectious mononucleosis ${ }^{12,13}$. Different cross reacting antigenic epitopes of non-typhoid organism like malaria TB and dengue are responsible for false positive reaction of slide Widal test.

Positive predictive value is the most important measure of a clinical diagnostic method since it represents the proportion of patient with positive result that are correctly diagnosed ${ }^{14}$. As in our study positive predictive value of slide test is very much poor; $70 \%$ it should not be used to detect true Enteric fever cases. However due to higher sensitivity (93.3\% sensitivity in our study) it can be used for rapid screening test and positive result should be confirmed by more specific $(98.57 \%$ specificity in our study) tube agglutination test otherwise due to false positivity many patient without enteric fever will be exposed to unnecessary antibiotic therapy leading to antibiotic resistance in future.

\section{CONCLUSIONS}

Slide Widal test has higher sensitivity but tube Widal test has higher specificity, so slide test should be used for screening purpose, but positive slide test result should be confirmed by tube Widal test.

\section{Limitations}

Our study was limited by small sample size. A large multicentred study including larger sample is necessary to come to a definitive conclusion.

\section{REFERENCES}

[1] Crump JA, Luby SP, Mintz ED. The global burden of typhoid fever. Bull World Health Organ 2004;82(5):34653.

[2] House D, Wain J, Ho VA, et al. Serology of typhoid fever in an area of endemicity and its relevance to diagnosis. J Clin Microbiol 2001;39(3):1002-7.

[3] Lavanya V, Solabannavar SS, Sonth SB. Comparison of results obtained by semi-quantitative slide agglutination and tube Widal tests in the diagnosis of suspected typhoid fever cases. Int J Biol Med Res 2013;4:3001-3.

[4] Bijapur GAM, Kakkerei SR, Raysa NP, et al. A study to determine significant titre-values of Widal test in the diagnosis of enteric fever for a population of North Kerala, India. Al Ameen J Med Sci 2014;7(1):72-7.

[5] Keddy KH, Sooka A, Letsoalo ME, et al. Sensitivity and specificity of typhoid fever rapid antibody tests for laboratory diagnosis at two sub-Saharan African sites. Bull World Health Organ 2011;89(9):640-7.

[6] Mandell G, Bennet J, Dolin R. Principles and practice of infectious disease. $7^{\text {th }}$ edn. Philadelphia, PA: Churchill Livingstone/Elsevier 2010.

[7] Oslen SJ, Pruckler J, Bibb W, et al. Evaluation of rapid diagnostic tests for typhoid fever. J Clin Microbiol 2004;42(5):1885-9.

[8] Wilke A. Ergonul O, Bayar B. Widal test in diagnosis of typhoid fever in Turkey. Clin Diag Lab Immunol 2002;9(4):938-41

[9] Das JC. Laboratory investigation of enteric fever in children: an update. J Chittagong Medical College Teachers' Association 2007;18(2):37-42.

[10] Welch H, Mickle LF. A rapid slide test for the serological diagnosis of typhoid and paratyphoid fevers. Am J Public Health Nations Health 1936;26(3):248-55.

[11] Handojo I, Edijianto SP, Probohoesodo MY, et al. Comparison of the diagnostic value of local Widal slide test with imported Widal slide test. Southeast Asian J Trop Med Public Health 2004;35(2):366-70.

[12] Radhika R, Subathra N. Comparison of tube Widal test and Slide Widal test in the diagnosis of Enteric Fever. Int J Microb Res 2017;9(4):888-90. 
[13] Jahan N, Khatoon R, Amrita, et al. Comparative evaluation of slide agglutination and Widal tube agglutination test in detecting enteric fever among patients attending a tertiary care hospital in North India. Int J Res Med Sci 2016;4(10):4290-6.
[14] Altman DG, Bland JM. Diagnostic tests 2: predictive values. BMJ 1994;309(6947):102. 